\title{
Root Handling Affects Carboxylates Exudation and Phosphate Uptake of White Lupin Roots
}

\author{
Raphael Tiziani ${ }^{*}$,Tanja Mimmo*, Fabio Valentinuzzi, Youry Pii, Silvia Celletti \\ and Stefano Cesco
}

Faculty of Science and Technology, Free University of Bolzano, Bolzano, Italy

OPEN ACCESS

Edited by: Gianpiero Vigani,

University of Turin, Italy

Reviewed by:

Enrico Martinoia,

University of Zurich, Switzerland

Agostino Sorgona,

Mediterranea University of Reggio

Calabria, Italy

*Correspondence:

Raphael Tiziani

Raphael.tiziani@natec.unibz.it

Tanja Mimmo

Tanja.mimmo@unibz.it

Specialty section: This article was submitted to

Plant Nutrition,

a section of the journal

Frontiers in Plant Science

Received: 17 July 2020

Accepted: 25 August 2020

Published: 02 October 2020

Citation:

Tiziani R, Mimmo T, Valentinuzzi $F$, Pii Y, Celletti S and Cesco S (2020) Root Handling Affects Carboxylates Exudation and Phosphate Uptake of White Lupin Roots.

Front. Plant Sci. 11:584568. doi: $10.3389 / \mathrm{fp} / \mathrm{s} .2020 .584568$
The reliable quantification of root exudation and nutrient uptake is a very challenging task, especially when considering single root segments. Most methods used necessitate root handling e.g. root dissecting/cutting. However, there is a knowledge gap on how much these techniques affect root physiology. Thus, this study aimed at assessing the effect of different root handling techniques on the phosphate $\left(\mathrm{P}_{\mathrm{i}}\right)$ uptake and carboxylate exudation of white lupin roots. White lupin plants were grown hydroponically in a full and $\mathrm{P}_{\mathrm{i}}$-deficient nutrient solution for 60 days. Phosphate uptake and carboxylate exudation of cluster and non-cluster roots were measured using custom made cells 1, 4, and $8 \mathrm{~h}$ after the onset of light. Three different experimental set-ups were used: i) without cutting the root apparatus from the shoots, nor dissecting the root into smaller root sections - named intact plant (IP); ii) separating the roots from the shoots, without dissecting the root into smaller sections - named intact root (IR); iii) separating the roots form the shoots and dissecting the roots in different sections - named dissected roots (DR). The sampling at $8 \mathrm{~h}$ led to the most significant alterations of the root $\mathrm{P}_{\mathrm{i}}$ uptake induced by the sampling method. Generally, roots were mainly affected by the DR sampling method, indicating that results of studies in which roots are cut/dissected should be interpreted carefully. Additionally, the study revealed that the root tip showed a very high $\mathrm{P}_{\mathrm{i}}$ uptake rate, suggesting that the tip could act as a $P_{\mathrm{i}}$ sensor. Citrate, malate and lactate could be detected in juvenile, mature and senescent cluster root exudation. We observed a significant effect of the handling method on carboxylate exudation only at sampling hours 1 and 8, although no clear and distinctive trend could be observed. Results here presented reveal that the root handling as well as the sampling time point can greatly influence root physiology and therefore should not be neglected when interpreting rhizosphere dynamics.

Keywords: carboxylates, cluster roots, phosphate uptake, root exudates, white lupin 


\section{INTRODUCTION}

Phosphorus (P) shortage is one of the most limiting factors for agricultural production (van de Wiel et al., 2016) due to the low concentration and poor mobility of its plant-available fraction in soils. Furthermore, P fertilization efficiency is lower than $30 \%$ highlighting the urgent need to study the physiological and molecular adaptations of plants to the limited endogenous $\mathrm{P}$ availability of soils. In addition, due to the limiting natural resources available to produce $\mathrm{P}$ fertilizers and the ever increasing demand of food for a growing population, it is urgent to improve P utilization efficiency (PUE) to maintain agricultural production at its maximal extent (Veneklaas et al., 2012; López-Arredondo et al., 2014). With respect to the plant effects, P shortage can lead to a reduced photosynthesis (Rodríguez et al., 1998; Wissuwa et al., 2005; Turnbull et al., 2007), an increased root-to-shoot ratio (Fredeen et al., 1989; López-Arredondo et al., 2014), an enhanced growth of lateral roots and root hairs (Reymond et al., 2006; Péret et al., 2011), a reduction in phosphorylated metabolites (Müller et al., 2015) and an increased expression of proteins that do not require $\mathrm{P}$ as co-substrates (Cruz-Ramírez et al., 2006; Gaude et al., 2008). One of the most important adaptation strategies of plants to $\mathrm{P}$ shortage is the increased release of root exudates into the rhizosphere. Root exudates are commonly defined as rootderived primary and secondary metabolites characterized by both low $(<1000 \mathrm{Da})$ and high $(>1000 \mathrm{Da})$ molecular weight (Oburger and Jones, 2018; Pausch and Kuzyakov, 2018). These compounds are exuded either actively (through primary or secondary active transporters) or passively (through diffusion and/or exocytosis processes, or thanks to ionic channels - Jones et al., 2009; Vives-Peris et al., 2019). With respect to their chemical nature, these root exudates consist of a very wide range of compounds including carbohydrates (e.g. glucose, fructose), amino acids (e.g. alanine, glycine, glutamate), carboxylates (e.g. malate, citrate, oxalate, lactate) or secondary metabolites such as phenols, glucosinolates, vitamins, plant hormones, but also enzymes (e.g. phosphatases, phytases) and polysaccharides (e.g. pectic acid). The type and concentration of metabolites exuded by roots depend on many factors such as i) the plant genotype, ii) the environment (e.g. soil properties, temperature etc.) and iii) biotic or abiotic stresses (Jones et al., 2009; Baetz and Martinoia, 2014; Steinauer et al., 2016; VivesPeris et al., 2019). The shortage of P mainly triggers the root release of phosphatases to mobilize the organic $\mathrm{P}$ pool, while carboxylates are mainly exuded in order to solubilize the nutrient from the inorganic fractions. In this regard, it is worth noting the role played by citrate and malate in root $\mathrm{P}$ acquisition by solubilizing and mobilizing via ligand exchange reactions the $\mathrm{P}$ bound to iron $(\mathrm{Fe})$ or aluminum (hydro)oxides (Otani et al., 1996; Neumann and Römheld, 1999).

White lupin (Lupinus albus L., Fabaceae family) is a nonmycorrhizal plant, which is well known to exude huge amounts of carboxylates, especially under P starvation (Mimmo et al., 2011; Marschner, 2012; Valentinuzzi et al., 2015). Therefore, this species serves often as a model plant in studies of root induced mobilization of sparingly soluble soil P sources (UhdeStone, 2017). Under P deficiency some plant species of the Proteaceae family develop special root types, the so-called proteoid roots (or cluster roots), which are short lateral rootlets with a bottlebrush-like appearance (Dinkelaker et al., 1989). These cluster roots are extremely efficient in enhancing soil $\mathrm{P}$ availability due to the increase of the roots surface area by more than 100 -fold and to the exudation of huge concentrations of protons, carboxylates and phosphatases, particularly in comparison with non-proteoid roots (Skene, 2000; Uhde-Stone, 2017). Furthermore, cluster roots also release large concentrations of flavonoids (e.g. genistein), probably to mobilize $\mathrm{P}$ complexed with $\mathrm{Fe}$ and to prevent microbial degradation of organic acids (Tomasi et al., 2008). It is interesting to note that the exudation of those compounds ensures the plant survival also in environments with basically no available P (Lambers et al., 2006). The Lupinus' cluster roots can be divided into distinct types based on their development stage: juvenile, immature, mature and senescent (Massonneau et al., 2001). The most active type of cluster roots in terms of carboxylate exudation is the mature one. Differently, the immature and juvenile rootlets are characterized for an abundant release of phenolic compounds (Weisskopf et al., 2006), whilst the senescent ones are mainly used for nutrient recycling (Massonneau et al., 2001; Wang et al., 2014). Moreover, there are some pieces of evidence showing that citrate exudation follows a diurnal and weekly pattern (Dessureault-Rompré et al., 2007; Tomasi et al., 2009; Mimmo et al., 2011). Even though the release of carboxylates has been widely described in literature, there is relatively limited information about the $\mathrm{P}$ uptake of the single cluster root types of white lupin. In this context, it is well known that $\mathrm{P}$-deficient plants generally upregulate phosphate $\left(\mathrm{P}_{\mathrm{i}}\right)$ transporters in order to cope with the nutritional disorder (Raghothama, 1999). Moreover, it seems that the P uptake rate in the cluster roots is plant species dependent. In fact, Malajczuk and Bowen (1974) revealed that Banskia grandis' cluster roots have faster $\mathrm{P}$ uptake rates when compared to their non-cluster roots. The same was observed in Protea compacta and Leucadendron uliginosum, which both showed faster $\mathrm{P}$ uptake rates in their cluster roots (Vorster and Jooste, 1986). On the other side, Banksia marginata seems to have a slower $\mathrm{P}$ uptake rate in cluster roots than in ordinary roots (Lamont, 1982). Interestingly, in Hakea prostata the P uptake of cluster roots was similar to that measured in non-cluster roots having the same age (Shane et al., 2004). In this regard, it is important to note that a better understanding of root exudation and $\mathrm{P}$ uptake phenomena is crucial to improve the crop performance especially in P-limited conditions in order to maximize yield. However, the reliable quantification of exudation and nutrient uptake is a very challenging task, particularly when planned at the same time and in more realistic conditions. Root exudate sampling methods are usually d4ivided into i) soil-based and ii) hydroponically-based methods (Oburger and Jones, 2018; Vives-Peris et al., 2019). Soil-based methods may lead to more realistic results, albeit much more difficult due to the 
complexity to access the roots and sampling of the rhizosphere, as well as due to the degradation of exudates by soil microflora. Hydroponically-based experiments may be easier to perform, but plant development, physiology and root exudation are often affected by hydroponic growing systems since they are highly artificial environments (Valentinuzzi et al., 2015). Both methods usually necessitate root handling and, thus, an effect of this processing on the root physiology cannot be totally excluded (Oburger and Schmidt, 2016; Oburger and Jones, 2018; Vives-Peris et al., 2019). Indeed, little is known about how much the root handling can influence the root exudation and $\mathrm{P}$ uptake phenomena. Moreover, up to now most studies have been performed by using excised cluster roots without an analysis of the impact of this cutting on the phenomenon studied. For this reason, we developed a custom-made device to analyze different hydroponically grown cluster root tissue types without the need to excise the single cluster rootlets. In particular, this study aimed at assessing the effect of different root handling techniques on the $\mathrm{P}$ uptake and carboxylate exudation of white lupin roots at three different time points during the day (i.e. 1, 4, and $8 \mathrm{~h}$ after the onset of light). Three different experimental set-ups have been used: i) without separating the roots from the shoots, ii) separating the roots from the shoots, iii) separating the roots from the shoots and dissecting the roots in the different sections.

\section{MATERIALS AND METHODS}

\section{Plant Growth}

Seeds of white lupin (Lupinus albus L. cv. Amiga; Südwestdeutsche Satzucht, Rastatt, Germany) were germinated for 5 to 6 days in the dark at $22^{\circ} \mathrm{C}$ between two layers of filter paper, which were moistened with $0.05 \mathrm{mmol} \mathrm{L}^{-1}$ $\mathrm{CaSO}_{4}$. Homogeneous seedlings were selected and placed in black pots, containing either a full (control, C) or a P-free (-P) nutrient solution (NS). The NS had the following composition: $0.25 \mathrm{mmol} \mathrm{L}^{-1} \mathrm{KH}_{2} \mathrm{PO}_{4}$ (not present in the $-\mathrm{P}$ solution), $5 \mathrm{mmol}$ $\mathrm{L}^{-1} \mathrm{Ca}\left(\mathrm{NO}_{3}\right)_{2} 4 \mathrm{H}_{2} \mathrm{O}, 1.25 \mathrm{mmol} \mathrm{L}^{-1} \mathrm{MgSO}_{4}, 1.75 \mathrm{mmol} \mathrm{L}^{-1}$ $\mathrm{K}_{2} \mathrm{SO}_{4}, 0.25 \mathrm{mmol} \mathrm{L}^{-1} \mathrm{KCl}, 20 \mu \mathrm{mol} \mathrm{L}{ }^{-1} \mathrm{Fe}(\mathrm{III})$-EDTA, $25 \mu \mathrm{mol}$ $\mathrm{L}^{-1} \mathrm{H}_{3} \mathrm{BO}_{4}, 1.25 \mu \mathrm{mol} \mathrm{L}-1 \mathrm{MnSO}_{4} 7 \mathrm{H}_{2} \mathrm{O}, 1.5 \mu \mathrm{mol} \mathrm{L}^{-1} \mathrm{ZnSO}_{4} 7$ $\mathrm{H}_{2} \mathrm{O}, 0.5 \mathrm{mmol} \mathrm{L}^{-1} \mathrm{CuSO}_{4} 5 \mathrm{H}_{2} \mathrm{O}, 0.025 \mu \mathrm{mol} \mathrm{L}{ }^{-1}\left(\mathrm{NH}_{4}\right)$ ${ }_{6} \mathrm{Mo}_{7} \mathrm{O}_{24} 4 \mathrm{H}_{2} \mathrm{O}$. The NS has been changed every 3 days. White lupin plants were grown in a climate chamber with the following conditions: day $14 \mathrm{~h}, 24^{\circ} \mathrm{C}, 70 \%$ relative humidity
(RH); night $10 \mathrm{~h}, 19^{\circ} \mathrm{C}, 70 \% \mathrm{RH}$. The experiments were executed after 60 days of growth, when the cluster roots of the plants were fully developed.

\section{Visualization of Acidification}

Qualitative visualizations of $\mathrm{pH}$ changes along the root axis were obtained using agar gel containing a $\mathrm{pH}$ indicator. Bromocresol purple $\left(0.1 \mathrm{~g} \mathrm{~L}^{-1}\right)$ adjusted to $\mathrm{pH} 6.2$ with $0.1 \mathrm{~mol} \mathrm{~L}^{-1} \mathrm{NaOH}$ was used as $\mathrm{pH}$ indicator. $1.8 \mathrm{~mL}$ of bromocresol purple was added in $500 \mathrm{~mL}$ liquid agar $(0.75 \%$, w/v). Selected roots were carefully placed in the $\mathrm{pH}$ indicator agar solution and incubated for $1 \mathrm{~h}$ at $25^{\circ} \mathrm{C}$ (Tomasi et al., 2013).

\section{Experimental Setup and Phosphate Uptake}

We developed a custom-made device to study root exudation and nutrient uptake with a spatial and temporal resolution of hydroponically grown plants roots (Figure 1). The device was 3D printed out of polylactic acid (CREA 3D S.R.L., Italy) with a Ultimaker 3D printer. The device was $24.4 \mathrm{~cm}$ long, $1.9 \mathrm{~cm}$ wide and consisted of $15(1.5 \mathrm{~cm}$ long x $1.5 \mathrm{~cm}$ wide) communicating cells (Figure 1). Roots of -P plants were divided in root apex (A), juvenile/immature cluster root $(\mathrm{J})$, mature cluster root $(\mathrm{M})$, senescent cluster roots $(\mathrm{S})$, root parts without cluster root $(\mathrm{R})$; whereas roots of $\mathrm{C}$ plants were divided into: roots parts without cluster (RC), cluster root like roots (C), control root apex (AC). We used three different experimental set-ups: i) without cutting the root apparatus from the shoots, nor dissecting the root into smaller root sections - named intact plant (IP); ii) separating the roots from the shoots, without dissecting the root into smaller sections- named intact root (IR); iii) separating the roots form the shoots and dissecting the roots in different sections-named dissected roots (DR) (Figure 2). In all the three different experimental set-ups, the roots were carefully placed inside the custom-made device in order to have the different root tissue segments as separated as possible. Previously, the roots were immerged in $0.05 \mathrm{mmol} \mathrm{L}^{-1} \mathrm{CaSO}_{4}$ for $15 \mathrm{~min}$ and dried carefully with tissue paper. Every cell of the custom-made device was filled with $3.4 \mathrm{~mL} 100 \mu \mathrm{mol} \mathrm{L}{ }^{-1} \mathrm{KH}_{2} \mathrm{PO}_{4}$ uptake/ root exudate collection solution. The roots were placed into the device and $\mathrm{P}_{\mathrm{i}}$ uptake and root exudation were measured. The use of radiolabeled $\mathrm{P}_{\mathrm{i}}$ was not possible since our laboratory is not allowed to use radioactive substances. The experiment was carried out three times during the day: 1,4 , and $8 \mathrm{~h}$ after the onset of light of the climate chamber. For all the three

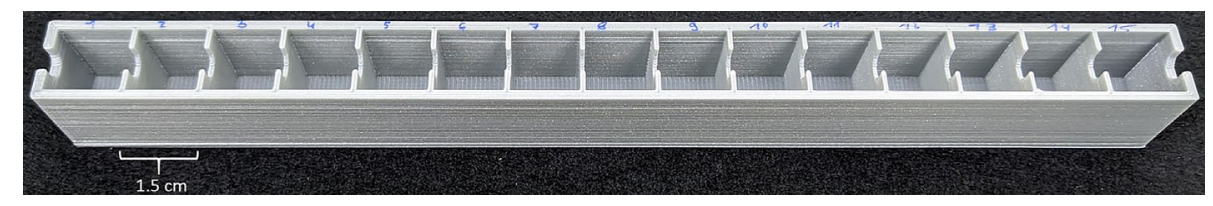

FIGURE 1 | Custom made 3D printed device to measure phosphate uptake and root exudation of single cluster root tissue types with a temporal and spatial resolution. 

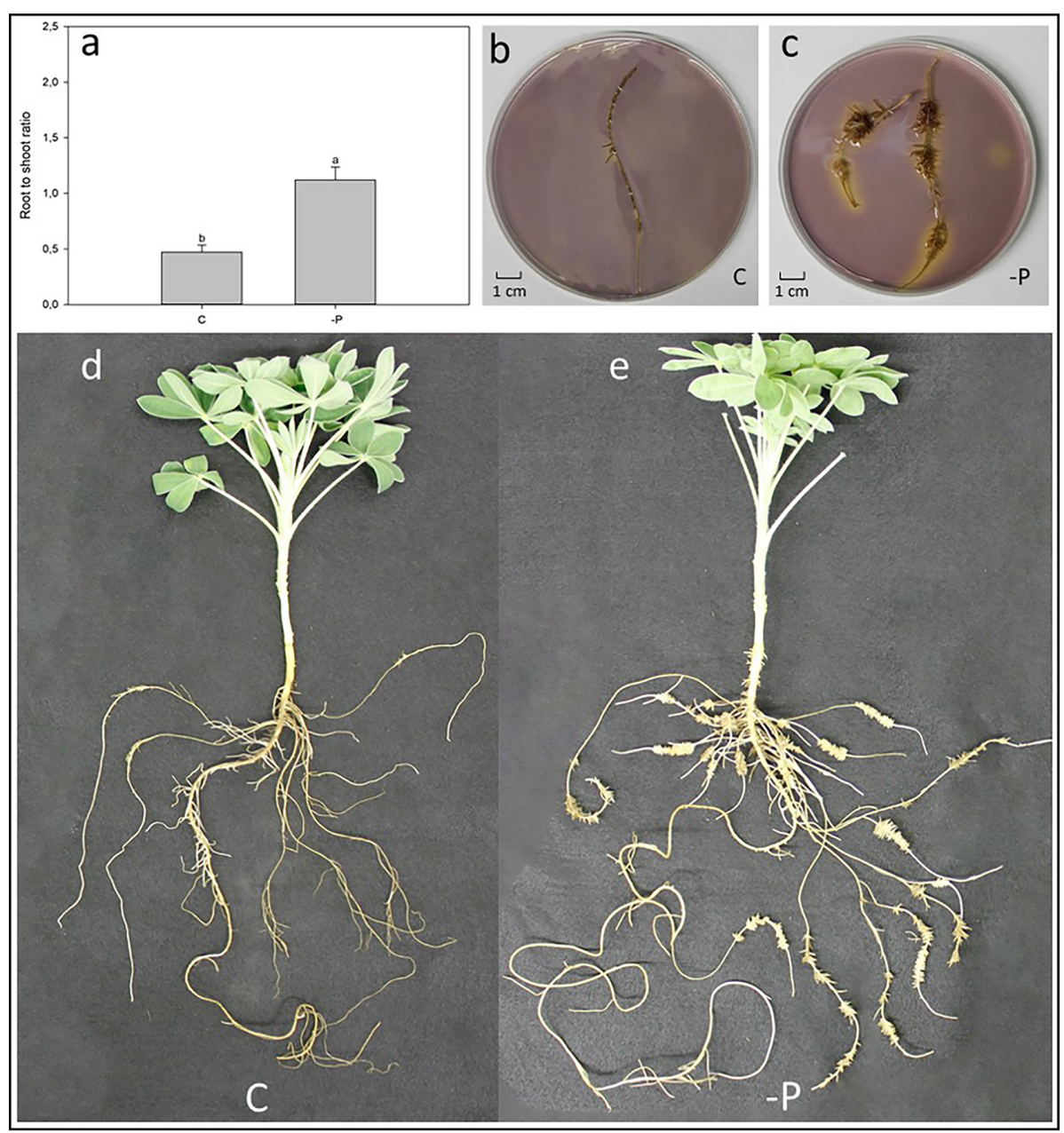

FIGURE 2 | Effect of P deficiency on 60-day-old white lupin plants. (A) Root-to-shoot ratio of white lupin plants grown in either control (C) or P starvation (-P) conditions; data are presented as mean \pm SE, $n=10$; lower case letters above the vertical bars indicate statistical significance according to $t$-test with $p<0.001$. (B) Acidification of a $\mathrm{C}$ white lupins' cluster root laid in an agar gel mixed with $\mathrm{pH}$ indicator: yellow coloring indicates a lower pH than the purple color.

(C) Acidification of a -P white lupins' root laid in an agar gel mixed with $\mathrm{pH}$ indicator: yellow coloring indicates a lower pH than the purple color. (D) Representative white lupin plant grown in $\mathrm{C}$ condition. (E) Representative white lupin plant grown in -P condition.

experimental set-ups and three sampling time points, we collected the root exudates after $1 \mathrm{~h}$ by pipetting $100 \mu \mathrm{l}$ of uptake solution directly in a HPLC vial for further analysis. Fifty $\mu \mathrm{l}$ uptake solution were sampled after 15,30 , and $60 \mathrm{~min}$ and their $\mathrm{P}_{\mathrm{i}}$ contents were analyzed. Phosphate uptake rate has been calculated by difference between the concentration of $\mathrm{PO}_{4}{ }^{3-}$ before and after the contact times of roots with the uptake solution. The values have been then normalized to the root fresh weight. The consumption of $\mathrm{P}_{\mathrm{i}}$ in the uptake medium due to the root uptake was always less than $10 \%$. After the collection of the solutions, the rootlets have been carefully dried with tissue paper and weighted. Phosphate concentration was determined colorimetrically according to the method of Murphy and Riley (1962). The method was scaled down in order to perform the reaction in a spectrophotometric cuvette with a final volume of 1 $\mathrm{mL}\left(50 \mu \mathrm{l}\right.$ of $\mathrm{KH}_{2} \mathrm{PO}_{4}$ standard, blank or sample, $5 \mu \mathrm{l} p$ - nitrophenol,10 $\mu \mathrm{l} 2.5 \mathrm{~mol} \mathrm{~L}^{-1}$ sulfuric acid $\left(\mathrm{H}_{2} \mathrm{SO}_{4}\right), 80 \mu \mathrm{l}$ of the Murphey and Riley reagent and $855 \mu$ l deionized water). The adsorption was read at $720 \mathrm{~nm}$ after $10 \mathrm{~min}$ incubation at room temperature.

\section{Analysis of Carboxylates by High- Performance Liquid Chromatography (HPLC)}

Carboxylates were separated on a cation exchange column (Aminex 87-H column, $300 \times 7.8 \mathrm{mmol} \mathrm{L}{ }^{-1}, 9 \mu \mathrm{m}$, Bio-Rad) using an isocratic elution with $10 \mathrm{mmol} \mathrm{L}^{-1} \mathrm{H}_{2} \mathrm{SO}_{4}$ as carrier solution at a flow rate of $0.6 \mathrm{~mL} \mathrm{~min}^{-1}$. Carboxylates were detected at $210 \mathrm{~nm}$ using a Waters 2998 photodiode array detector (Waters Spa, Italy). Standard carboxylates were prepared as individual stock solutions, using Sigma reagent grade compounds, and then combined to give diluted reference standards. Comparing the 
retention times of unknown compounds with those of pure compounds allowed the detection of carboxylates.

\section{Statistical Analysis}

The results are presented as means \pm standard error (SE). Statistical analysis was performed using SigmaPlot 12 on Windows 1064 bit. Two conditions were compared through ttests; p-values of $<0.05$ were treated as statistically significant differences. Three and more conditions were analyzed by oneway analysis of variance (ANOVA) and means were compared using Holm-Sidak post hoc test at $\mathrm{p}<0.05$ to determine the significance of found differences.

\section{RESULTS}

\section{Root Morphology and Acidification}

Figure 3A shows the root-to-shoot ratio of hydroponically grown white lupin plants, raised in control (C) and $\mathrm{P}$ deficiency (-P) conditions after 60 days. As expected, -P lupin plants showed a significantly higher $(+42 \%)$ root-to-shoot ratio compared to $\mathrm{C}$ plants. Indeed, white lupin plants grown in $-\mathrm{P}$ developed huge amounts of cluster roots (Figure 3E), while plants grown control solution exhibited a much smaller root apparatus developing only very small amounts of cluster roots like root structures (Figure 3D). Furthermore, cluster roots of Pdeficient plants acidified the rhizosphere as shown by the yellow color around the $-\mathrm{P}$ roots (Figure 3C). On the other hand, roots from white lupin plants grown in a full NS showed just a very limited acidification of the rhizosphere when compared to -P grown white lupin roots (Figure 3B).

\section{Phosphate Uptake}

\section{Phosphate Uptake After $1 \mathrm{~h}$}

Table 1 shows the $\mathrm{P}_{\mathrm{i}}$ uptake rate of $\mathrm{C}$ and -P roots $1 \mathrm{~h}$ after the onset of light in the climate chamber. The three different root handlings (IP, IR, DR) did not influence the $\mathrm{P}_{\mathrm{i}}$ uptake in any of the two growing conditions (-P and $\mathrm{C}$ ). On the other side, the $\mathrm{P}_{\mathrm{i}}$ uptake was significantly affected by the different root segments within the same handling methods. In the intact plant (IP) handling method, the apex of -P roots (A) showed the highest uptake rate, while the lowest was found in mature cluster roots of -P plants (M) (A vs $\mathrm{M}+299 \%)$. In the handling method in which the roots were detached from the shoot but kept intact (IR), the significantly highest $\mathrm{P}_{\mathrm{i}}$ uptake rate was found unexpectedly in apex rootlets of control plants (AC) and apex rootlets of -P lupins (A). On average the AC and A roots showed a $266 \%$ higher uptake when compared to all the other rootlets of both control and P-deficient plants. A similar trend could be observed when considering the completely dissected roots (DR) handling method. When the roots were completely dissected, the highest $\mathrm{P}_{\mathrm{i}}$ uptake was found in $\mathrm{AC}, \mathrm{A}$, and RC rootlets.

\section{Phosphate Uptake After $4 \mathrm{~h}$}

Table 2 shows the $\mathrm{P}_{\mathrm{i}}$ uptake of $-\mathrm{P}$ and $\mathrm{C}$ white lupin roots $4 \mathrm{~h}$ after the onset of the light. It is interesting to note significant differences just in roots belonging to white lupins grown in a full nutrient solution (C), with all three root handlings (IP, IR, DR). In $A C$ rootlets, the significant highest $P_{i}$ uptake rate was detected when roots were dissected into single segments prior to the uptake experiment (DR), while it resulted lowest when the roots were separated from the plants without cutting them into smaller pieces (IR, Table 2). A similar trend could be observed in cluster rootlets C plants. Again, the single dissected root segments (DR) revealed the highest $\mathrm{P}_{\mathrm{i}}$ uptake rate compared to roots sampled with the other two root handling set-ups (DR vs IR + 273\%; DR vs IP $+826 \%)$. The $\mathrm{P}_{\mathrm{i}}$ uptake rate in $\mathrm{RC}$ rootlets showed the same tendency: $\mathrm{P}_{\mathrm{i}}$ uptake rate resulted highest in dissected root segments (DR vs IR +337\%; DR vs IP + 283\%). When looking at the differences among the root tissues types, only the intact roots (IR) handling method displayed significant differences. The $\mathrm{P}_{\mathrm{i}}$ uptake rate was the highest in $\mathrm{R}$ rootlets, together with $\mathrm{A}$ and $\mathrm{RC}$ rootlets. On average they showed a $287 \%$ higher $\mathrm{P}_{\mathrm{i}}$ uptake rate compared to all the other root tissues (J, M, S, AC, C).

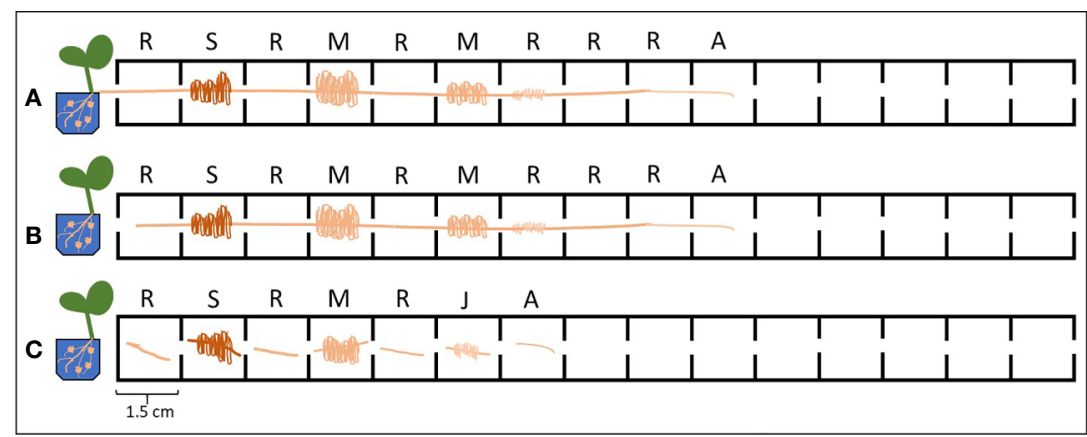

FIGURE 3 | Different experimental set-ups used for the $\mathrm{P}_{\mathrm{i}}$ uptake/exudation experiment. Letters above the cells indicate the root tissue type: $\mathrm{R}$, root without cluster; S, senescent cluster root; M, mature cluster root; J, juvenile cluster root; A, apex. (A) Intact plants (IP): without cutting the root apparatus from the shoots nor dissecting the root into smaller root sections. (B) Intact roots (IR): separating the roots from the shoots, without dissecting the root into smaller root sections. (C) Dissected roots (DR): separating the roots from the shoots and dissecting the roots in different sections. 


\section{$-\mathrm{P}$ root}

\section{C root}
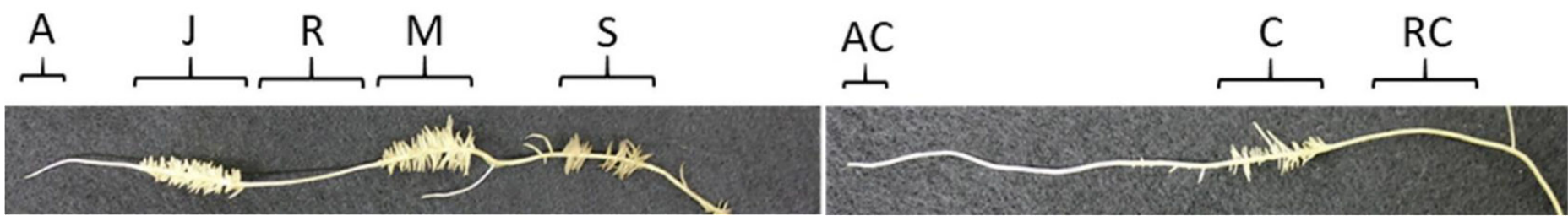

1h

Sampling

Root segment

\begin{tabular}{|c|c|c|c|c|c|c|c|c|c|c|}
\hline & & A & $\mathbf{J}$ & $\mathbf{R}$ & M & $\mathbf{s}$ & AC & C & RC & $p$ \\
\hline$P_{\mathrm{i}}$ uptake $\left(\mu \mathrm{mol} \mathrm{g}^{-1}\right.$ & $\mathbb{P}$ & $9.03 \pm 3.69 a \mathrm{~A}$ & $4.18 \pm 1.5 \mathrm{abc} A$ & $5.15 \pm 1.1 \mathrm{abc} A$ & $2.26 \pm 0.56 c \mathrm{~A}$ & $2.55 \pm 0.77 \mathrm{bc} \mathrm{A}$ & $5.66 \pm 5.3 \mathrm{abc} A$ & $2.46 \pm 0.85 b c A$ & $7.40 \pm 1.24 a b$ A & $<0.001$ \\
\hline \multirow[t]{2}{*}{$\mathrm{FW} \mathrm{h}^{-1}$ ) } & $\mathbb{I R}$ & $14.33 \pm 4.1 \mathrm{ab} \mathrm{A}$ & $0.92 \pm 0.25 \mathrm{~cd} \mathrm{~A}$ & $8.73 \pm 1.96 \mathrm{bc} \mathrm{A}$ & $2.50 \pm 0.69 \mathrm{~cd} A$ & $0.79 \pm 0.24 d A$ & $22.7 \pm 1.33 \mathrm{a} A$ & $7.84 \pm 5 b c d A$ & $9.53 \pm 1.86 \mathrm{~b} \mathrm{~A}$ & $<0.001$ \\
\hline & DR & $7.96 \pm 0.97 \mathrm{ab} A$ & $2.29 \pm 0.72 b c$ A & $6.80 \pm 0.43 b \mathrm{~b}$ & $1.21 \pm 0.20 \mathrm{c} \mathrm{A}$ & $2.01 \pm 0.61 b c A$ & $13.51 \pm 4.12 \mathrm{a} \mathrm{A}$ & $4.64 \pm 0.76 b c \mathrm{~A}$ & $10.78 \pm 1.27 \mathrm{a} \mathrm{A}$ & $<0.001$ \\
\hline$p$ & & ns & ns & ns & ns & ns & ns & ns & ns & \\
\hline Citrate $(\mathrm{nmol} \mathrm{g})^{-1}$ & IP & - & $3.24 \pm 0.20 \mathrm{a} A$ & - & $2.02 \pm 0.06 \mathrm{c} B$ & $2.64 \pm 0.19 b \mathrm{~A}$ & - & - & - & 0.002 \\
\hline \multirow[t]{2}{*}{$\mathrm{FW} \mathrm{h}^{-1}$ ) } & $\mathbb{R}$ & - & $3.52 \pm 0.23 a \mathrm{~A}$ & - & $2.88 \pm 0.04 a \mathrm{~A}$ & $3.66 \pm 0.97 \mathrm{a} A$ & - & - & - & ns \\
\hline & $\mathrm{DR}$ & - & $2.55 \pm 0.22 \mathrm{ab} B$ & - & $3.39 \pm 0.45 a \mathrm{~A}$ & $1.49 \pm 0.23 b \mathrm{~A}$ & - & - & - & 0.027 \\
\hline$p$ & & & 0.0047 & & 0.043 & ns & & & & \\
\hline Malate $(\mathrm{nmol} \mathrm{g})^{-1}$ & $\mathbb{I P}$ & - & $2.09 \pm 0.09 a \mathrm{~A}$ & - & $1.42 \pm 0.10 \mathrm{~b} \mathrm{~A}$ & $2.00 \pm 0.15 a \mathrm{~A}$ & - & - & - & 0.003 \\
\hline \multirow[t]{2}{*}{$\mathrm{FW} \mathrm{h}^{-1}$ ) } & $\mathbb{R}$ & - & $1.58 \pm 0.06 \mathrm{a} \mathrm{A}$ & - & $1.21 \pm 0.06 \mathrm{a} A$ & $0.90 \pm 0.21 a A$ & - & - & - & ns \\
\hline & $\mathrm{DR}$ & - & $1.38 \pm 0.34 \mathrm{a} \mathrm{A}$ & - & $1.25 \pm 0.07 \mathrm{a} \mathrm{A}$ & $0.56 \pm 0.08 \mathrm{a} B$ & - & - & - & ns \\
\hline$p$ & & & ns & & ns & $<0.001$ & & & & \\
\hline Lactate $\left(\mathrm{nmol} \mathrm{g}{ }^{-1}\right.$ & IP & - & $2.54 \pm 0.17 \mathrm{a} \mathrm{A}$ & - & $2.85 \pm 0.09 a \mathrm{~A}$ & $2.54 \pm 0.23 a \mathrm{~A}$ & - & - & - & ns \\
\hline \multirow[t]{2}{*}{$\mathrm{FW} \mathrm{h}^{-1}$ ) } & $\mathbb{R}$ & - & $2.51 \pm 0.14 \mathrm{a} \mathrm{A}$ & - & $1.62 \pm 0.19 b \mathrm{~B}$ & $1.45 \pm 0.13 b$ B & - & - & - & 0.006 \\
\hline & $\mathrm{DR}$ & - & $2.51 \pm 0.24 \mathrm{a} A$ & - & $1.38 \pm 0.03 b \mathrm{~b}$ & - & _- & _- & _- & 0.01 \\
\hline$p$ & & & ns & & $<0.001$ & 0.014 & & & & \\
\hline
\end{tabular}

Phosphate uptake rate has been calculated by difference between the concentration of $\mathrm{PO}_{4}{ }^{3-}$ before and after the contact times of roots with the uptake solution. -P roots have been separated in five different root segments: $A$, apex; J, juvenile cluster root; $R$, root; $M$, mature cluster root; $S$, senescent cluster root; $C$ roots have been separated in three different root segments: $A C$, apex control; $C$, cluster control; RC, root control. Phosphate uptake and carboxylate exudation have been determined using three different root handlings: IP, intact plants; IR, intact roots; $D R$, dissected roots. Data are presented as mean $\pm S E, n=5$. Lower case letters indicate statistical significance between different root segments (i.e. A vs $J$ ) within the same root handling method according to one-way ANOVA comparing means by Holm-Sidak post hoc test with a significance threshold at $p<0.05$. Upper case letters indicate statistical significance within the same root segments but different sampling methods (i.e. IP vs IR) according to one-way ANOVA comparing means by Holm-Sidak post hoc test with a significance threshold at $p<0.05$; ns, non-significant. 


\section{-P root}

\section{C root}
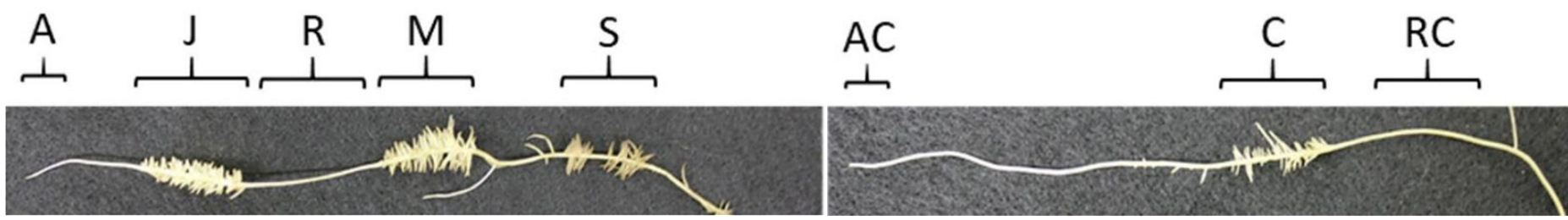

4h

Sampling

Root segment

\begin{tabular}{|c|c|c|c|c|c|c|c|c|c|c|}
\hline & & A & $\mathbf{J}$ & $\mathbf{R}$ & M & $\mathbf{s}$ & $A C$ & c & RC & $p$ \\
\hline$P_{\mathrm{i}}$ uptake $\left(\mu \mathrm{mol} \mathrm{g}^{-1}\right.$ & $\mathbb{P}$ & $0.89 \pm 0.12 \mathrm{a} A$ & $2.79 \pm 1.09 a \mathrm{~A}$ & $0.58 \pm 0.32 \mathrm{a} A$ & $1.57 \pm 0.14 \mathrm{a} A$ & $0.94 \pm 0.16 a \mathrm{~A}$ & $5.49 \pm 0.68 a \mathrm{~B}$ & $0.60 \pm 0.19 a \mathrm{~B}$ & $2.83 \pm 1.5 \mathrm{a} B$ & ns \\
\hline \multirow[t]{2}{*}{$\mathrm{FW} \mathrm{h}^{-1}$ ) } & $\mathbb{R}$ & $3.54 \pm 0.61 \mathrm{a} A$ & $1.75 \pm 0.41 b c \mathrm{~A}$ & $3.75 \pm 0.75 a \mathrm{~A}$ & $1.09 \pm 0.30 \mathrm{bc} \mathrm{A}$ & $0.51 \pm 0.14 \mathrm{c} \mathrm{A}$ & $0.76 \pm 0.14 c \mathrm{C}$ & $1.49 \pm 0.32 b c B$ & $2.48 \pm 0.57 a b$ B & $<0.001$ \\
\hline & $\mathrm{DR}$ & $6.79 \pm 2.96 a \mathrm{~A}$ & $4.58 \pm 1.21 \mathrm{a} A$ & $3.51 \pm 0.98 a \mathrm{~A}$ & $1.61 \pm 0.42 \mathrm{a} A$ & $1.11 \pm 0.36 a \mathrm{~A}$ & $21.14 \pm 2.11 \mathrm{a} \mathrm{A}$ & $5.56 \pm 1.45 a \mathrm{~A}$ & $10.85 \pm 2.24 a \mathrm{~A}$ & ns \\
\hline$p$ & & $\mathrm{~ns}$ & ns & $\mathrm{ns}$ & ns & ns & $<0.001$ & 0.017 & 0.017 & \\
\hline Citrate $\left(\mathrm{nmol} \mathrm{g}{ }^{-1}\right.$ & $\mathbb{P}$ & - & $6.90 \pm 0.84 a \mathrm{~A}$ & - & $6.67 \pm 0.36 a \mathrm{~A}$ & $3.49 \pm 0.16 b \mathrm{~A}$ & - & - & - & 0.002 \\
\hline \multirow[t]{2}{*}{$\mathrm{FW} \mathrm{h}^{\mathrm{v}-1}$ ) } & $\mathbb{R}$ & - & $5.60 \pm 0.59 a \mathrm{~A}$ & - & $4.92 \pm 0.42 \mathrm{a} \mathrm{A}$ & $3.32 \pm 0.58 \mathrm{a} A$ & - & - & - & ns \\
\hline & DR & - & $5.91 \pm 1.13 a \mathrm{~A}$ & - & $6.58 \pm 0.66 a \mathrm{~A}$ & $4.38 \pm 0.73 a \mathrm{~A}$ & - & - & - & ns \\
\hline$p$ & & & ns & & ns & ns & & & & \\
\hline Malate $\left(\mathrm{nmol} \mathrm{g}{ }^{-1}\right.$ & $\mathbb{I P}$ & - & $1.67 \pm 0.31 \mathrm{~b} \mathrm{~A}$ & - & $2.50 \pm 0.37 \mathrm{~b} \mathrm{~A}$ & $5.70 \pm 0.32 a \mathrm{~A}$ & - & - & - & 0.003 \\
\hline \multirow{3}{*}{$\mathrm{FW} \mathrm{h}^{-1}$ ) } & $\mathbb{R}$ & - & $2.84 \pm 0.70 \mathrm{a} A$ & - & $1.67 \pm 0.34 a \mathrm{~A}$ & $2.99 \pm 0.19 a \mathrm{~B}$ & - & - & - & ns \\
\hline & DR & - & $4.08 \pm 0.86 a \mathrm{~A}$ & - & $2.23 \pm 0.46 a \mathrm{~A}$ & $1.69 \pm 0.07 a \mathrm{C}$ & - & - & - & ns \\
\hline & & & ns & & ns & $<0.001$ & & & & \\
\hline Lactate $\left(\mathrm{nmol} \mathrm{g}{ }^{-1}\right.$ & $\mathbb{P}$ & - & $3.93 \pm 1.04 a \mathrm{~A}$ & - & $6.02 \pm 1.34 \mathrm{a} A$ & $3.70 \pm 0.88 a \mathrm{~A}$ & - & - & - & ns \\
\hline \multirow[t]{3}{*}{$\left.F W h^{-1}\right)$} & $\mathbb{R}$ & - & $4.54 \pm 0.88 a \mathrm{~A}$ & - & $3.72 \pm 1.14 \mathrm{ab} A$ & $2.17 \pm 0.54 b \mathrm{~A}$ & - & - & - & 0.006 \\
\hline & DR & - & $4.72 \pm 1.16 \mathrm{a} \mathrm{A}$ & - & $2.94 \pm 0.28 b \mathrm{~A}$ & - & - & - & - & 0.01 \\
\hline & & & ns & & ns & ns & & & & \\
\hline
\end{tabular}

Phosphate uptake rate has been calculated by difference between the concentration of $\mathrm{PO}_{4}{ }^{3-}$ before and after the contact times of roots with the uptake solution. -P roots have been separated in five different root segments: $A$, apex; J, juvenile cluster root; R, root; M, mature cluster root; S, senescent cluster root. C roots have been separated in three different root segments: AC, apex control; C, cluster control; RC, root control. Phosphate uptake and carboxylate exudation have been determined using three different root handlings: IP, intact plants; IR, intact roots; $D R$, dissected roots. Data are presented as mean $\pm S E, n=5$. Lower case letters indicate statistical significance between different root segment (i.e. A vs $\mathrm{J}$ ) within the same root handling method according to one-way ANOVA comparing means by Holm-Sidak post hoc test with a significance threshold at $p<0.05$. Upper case letters indicate statistical significance within the same root segment but different sampling methods (i.e. IP vs IR) according to one-way ANOVA comparing means by Holm-Sidak post hoc test with a significance threshold at $p<0.05$; ns, non-significant. 


\section{$-\mathrm{P}$ root}

\section{C root}
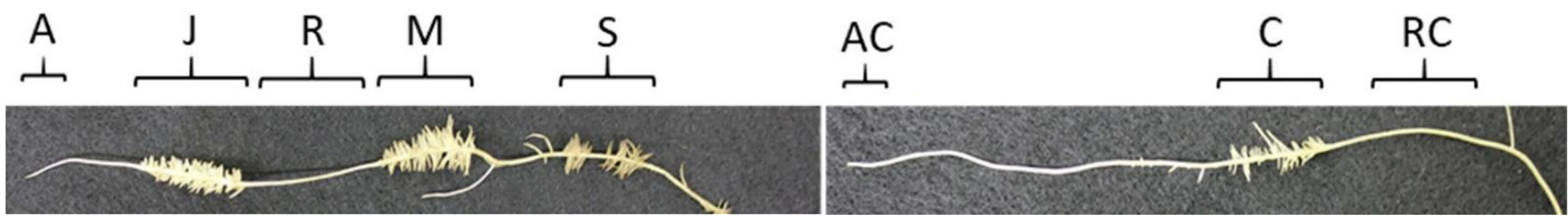

Sampling

Root segment

\begin{tabular}{|c|c|c|c|c|c|c|c|c|c|c|}
\hline & & A & $J$ & $\mathbf{R}$ & M & s & $A C$ & C & RC & $p$ \\
\hline $\mathrm{P}_{\mathrm{i}}$ uptake $(\mu \mathrm{mol}$ & $\mathbb{P}$ & $10.94 \pm 6 \mathrm{ab} A$ & $0.37 \pm 0.22 \mathrm{ab} A$ & $12.33 \pm 3.68 \mathrm{a} A$ & $1.66 \pm 0.40 \mathrm{ab} B$ & $0.34 \pm 0.07 \mathrm{~b} B$ & $8.93 \pm 0.65 \mathrm{ab} A$ & $6.29 \pm 2.9 \mathrm{ab} A B$ & $3.93 \pm 2.1 \mathrm{ab} A B$ & 0.013 \\
\hline \multirow[t]{2}{*}{$\left.\mathrm{g}^{-1} \mathrm{FW}\right)$} & $\mathbb{R}$ & $1.48 \pm 0.37 a \mathrm{~B}$ & $0.54 \pm 0.17 \mathrm{a} A$ & $3.05 \pm 0.59 a \mathrm{~B}$ & $0.64 \pm 0.15 a \mathrm{~B}$ & $0.45 \pm 0.04 a \mathrm{~B}$ & $0.63 \pm 0.47 \mathrm{a} C$ & $1.22 \pm 0.56 a \mathrm{~B}$ & $2.36 \pm 0.93 a \mathrm{~B}$ & ns \\
\hline & DR & $12.63 \pm 0.79 a \mathrm{~A}$ & $1.39 \pm 0.36 a \mathrm{~A}$ & $1.73 \pm 0.79 a C$ & $2.53 \pm 0.44 a \mathrm{~A}$ & $2.08 \pm 0.73 a \mathrm{~A}$ & $3.32 \pm 0.07 a \mathrm{~B}$ & $7.71 \pm 3.70 \mathrm{a} A$ & $9.37 \pm 2.28 \mathrm{a} A$ & ns \\
\hline$p$ & & 0.013 & ns & $<0.001$ & 0.009 & 0.009 & $<0.001$ & 0.023 & 0.023 & \\
\hline Citrate & IP & - & $3.88 \pm 0.47 a \mathrm{~B}$ & - & $2.24 \pm 0.45 b \mathrm{~B}$ & $1.72 \pm 0.24 b B$ & - & - & - & 0.002 \\
\hline \multirow{2}{*}{$\left(\mathrm{nmol} \mathrm{g}{ }^{-1} \mathrm{FW} \mathrm{h} \mathrm{h}^{\mathrm{v}}\right)$} & $\mathbb{R}$ & - & $3.34 \pm 0.45 a \mathrm{~B}$ & - & $2.81 \pm 0.24 a \mathrm{~B}$ & $3.85 \pm 0.58 a A B$ & - & - & - & ns \\
\hline & DR & - & $6.39 \pm 0.32 a \mathrm{~A}$ & - & $4.39 \pm 0.12 b \mathrm{~b}$ & $4.74 \pm 0.49 a b \mathrm{~A}$ & - & - & - & 0.027 \\
\hline$p$ & & & 0.013 & & 0.002 & 0.024 & & & & \\
\hline Malate $\left(\mathrm{nmol} \mathrm{g}^{-1}\right.$ & $\mathbb{P}$ & - & $3.27 \pm 0.75 a \mathrm{~A}$ & - & $1.51 \pm 0.25 b \mathrm{~A}$ & $1.59 \pm 0.47 \mathrm{~b} \mathrm{~A}$ & - & - & - & 0.003 \\
\hline \multirow[t]{2}{*}{$\mathrm{FW} \mathrm{h}^{-1}$ ) } & $\mathbb{R}$ & - & $2.04 \pm 0.38 a \mathrm{~A}$ & - & $1.09 \pm 0.28 a \mathrm{~A}$ & $1.37 \pm 0.13 a \mathrm{~A}$ & - & - & - & ns \\
\hline & DR & - & $3.58 \pm 1.16 \mathrm{a} A$ & - & $1.53 \pm 0.17 \mathrm{a} \mathrm{A}$ & - & - & - & - & ns \\
\hline$p$ & & & ns & & ns & ns & & & & \\
\hline Lactate $\left(\mathrm{nmol} \mathrm{g}{ }^{-1}\right.$ & IP & - & $5.14 \pm 0.44 a \mathrm{~A}$ & - & $2.47 \pm 0.34 \mathrm{~b} \mathrm{~A}$ & $2.47 \pm 0.96 b \mathrm{~b}$ & - & - & - & ns \\
\hline \multirow[t]{2}{*}{$\left.F W h^{-1}\right)$} & $\mathbb{R}$ & - & $2.47 \pm 0.52 a \mathrm{~A}$ & - & $2.32 \pm 0.47 a \mathrm{~A}$ & $3.07 \pm 0.95 \mathrm{a} A$ & - & - & - & ns \\
\hline & DR & - & $5.06 \pm 0.57 \mathrm{a} \mathrm{A}$ & - & $2.77 \pm 0.34 b \mathrm{~A}$ & - & - & - & - & 0.01 \\
\hline$p$ & & & ns & & ns & ns & & & & \\
\hline
\end{tabular}$$
\text { ns }
$$

Phosphate uptake rate has been calculated by difference between the concentration of $P_{A_{4}-}^{3-}$ before and after the contact times of roots with the uptake solution. -P roots have been separated in 5 different root segments: $A=$ apex, $J=$ juvenile cluster root, $R=$ root, $M=$ mature cluster root, $S=$ senescent cluster root. C roots have been separated in 3 different root segments: $A C=$ apex control, $C=$ cluster control, $R C=$ root control. Phosphate uptake and carboxylate exudation have been determined using three different root handlings: $I P=$ intact plants, $I R=$ intact roots, $D R=$ dissected roots. Data are presented as mean $\pm S E, n=5$. Lower case letters indicate statistical significance between different root segments (i.e. $A$ vs J) within the same root handling method according to one-way ANOVA comparing means by Holm-Sidak post hoc test with a significance threshold at $p<0.05$. Upper case letters indicate statistical significance within the same root segment but different sampling methods (i.e. IP vs IR) according to one-way ANOVA comparing means by Holm-Sidak post hoc test with a significance threshold at $p<0.05$; $n s=$ non-significant. 


\section{Phosphate Uptake After $8 \mathrm{~h}$}

Table 3 reports the $P_{i}$ uptake of white lupin rootlets $8 \mathrm{~h}$ after the onset of light. The experimental set-up had a greater effect on the $\mathrm{P}_{\mathrm{i}}$ uptake at this time point. Besides the J cluster roots, all root tissue types were significantly affected by the root handling in both full and $\mathrm{P}$-deficient nutrient status. In -P conditions, dissected root apices (DR, roots type: A) displayed a $753 \%$ higher uptake rate when compared to the apices of the whole root separated from the shoots (IR). The same trend could be observed in mature cluster roots (M roots: $+295 \%$ DR vs IR; $+52.4 \%$ DR vs IP). Furthermore, also senescent rootlets (S) presented the significantly highest $\mathrm{P}$ uptake rate in the dissected root segments (IR) having a 362\% and 517\% higher rate compared to entire roots separated from the shoots (IR) and the roots from intact plants (IP), respectively. The $\mathrm{R}$ root tissue type of -P lupin plants displayed the opposite trend showing the highest uptake rate when root segments were not excised (IP and IR experimental set-ups: $+304 \%$ IP $v s$ DR; $+74 \%$ IR $v s$ DR). The apices of control white lupin plants (AC) exhibited a similar $\mathrm{P}_{\mathrm{i}}$ uptake pattern: apices of intact plants showed the highest $\mathrm{P}_{\mathrm{i}}$ uptake rate (+1317\% IP vs IR; $+168 \%$ IP vs DR). On the other hand, $\mathrm{P}_{\mathrm{i}}$ uptake resulted highest in $\mathrm{C}$ rootlets of the excised root segments (+531\% DR $v s$ IR). Again, RC rootlets exhibited a $297 \%$ higher $\mathrm{P}$ uptake rate when the roots were dissected in segments rather than kept as entire roots (DR vs IR). Concerning the effect on the $\mathrm{P}_{\mathrm{i}}$ uptake of the root tissue types, significant differences could be observed only in the sampling method in which the plants were kept intact (IP). The significantly highest uptake rate was discovered in the $\mathrm{R}$ tissue type (-P plants), the lowest in the $\mathrm{S}$ tissue type (-P plants, $\mathrm{R} v s \mathrm{~S}+3626 \%$ ). All the other root zones (A, J, M, AC, C, RC) were between those two without any statistical significance.

\section{Release of Carboxylates}

\section{Release of Carboxylates After $1 \mathrm{~h}$}

Table 1 reports the carboxylate exudation of both $\mathrm{C}$ and -P lupin plants detected in the collection solution of the different root zones after $1 \mathrm{~h}$ from the onset of light. Carboxylate analysis revealed the presence of citrate, malate and lactate in the collection solution of white lupin roots. Citrate, malate and lactate have been detected only in juvenile (J), mature (M) and senescent (S) roots of -P plants.

Citrate exudation of $\mathrm{J}$ and $\mathrm{M}$ roots was affected by the type of root handling (Table 1). Juvenile roots exhibited a significantly higher exudation rate when roots were not dissected in segments (+32.5\% IP and IR vs DR). On the contrary, citrate release from $\mathrm{M}$ roots was highest when roots were either dissected in segments or separated from the shoots $(+67.3 \%$ DR vs IP; + $42.5 \%$ IR vs IP). Citrate exudation from $S$ roots was not significantly influenced by the root handling method. When comparing the citrate exudation of the tissue types, significant variations were found in the IP and DR handling method. In the first one J cluster roots showed the highest release of citrate, followed by $\mathrm{S}$ roots and finally $\mathrm{M}$ cluster roots. In the handling method in which the roots were dissected completely into pieces prior to the sampling (DR), the highest citrate release was observed in $\mathrm{M}$ rootlets compared to $\mathrm{S}$ rootlets ( $\mathrm{M} v s \mathrm{~S}+227 \%$ ).

Interestingly, $S$ roots revealed a root handling-dependent exudation pattern for malate (Table 1). Indeed, malate exudation resulted highest when roots were not excised in segments: $S$ roots exhibited a 4 -fold and 2-fold higher malate exudation rate from intact plants (IP) and roots separated from their shoots (IR), respectively, compared to the dissected $S$ roots (DR). Moreover, concerning the differences of the root tissue types only $\mathrm{S}$ and $\mathrm{J}$ rootlets displayed a significant higher malate exudation compared to $\mathrm{M}$ roots, yet only in the IP root handling method. On average $\mathrm{S}$ and J roots showed a $44 \%$ higher malate exudation rate compared to $M$ rootlets.

Lactate revealed a completely different pattern compared to citrate and malate (Table 1). In particular, $M$ roots released higher lactate rates when roots were not dissected nor separated from their shoots $(+75.9 \%$ IP $v s$ IR, $+106 \%$ IP $v s$ DR). Additionally, S roots showed a significant $75.2 \%$ higher lactate exudation rate from roots of the intact plant (IP) when compared to the roots which were separated from their shoots (IR). It is interesting to note that lactate was not detected in excised $S$ roots (Table 1). Regarding the changes of lactate exudation among different root tissue types significant differences could be found in the two handling methods in which the roots were cut (IR, DR). In both cases, the significantly highest lactate release was observed in J roots compared to $\mathrm{M}$ and $\mathrm{S}$ roots (IR: $+53 \% \mathrm{~J}$ vs $\mathrm{M} / \mathrm{S} ;+81 \%$ DR: J vs $\mathrm{M})$.

\section{Release of Carboxylates After $4 \mathrm{~h}$}

Table 2 reports the carboxylate exudation detected in the collection solution of the different root segments after $4 \mathrm{~h}$ from onset of light of the climate chamber of both white lupin plants raised in $\mathrm{C}$ and $-\mathrm{P}$ nutrient conditions. Analysis of the carboxylates revealed again the presence of citrate, malate, and lactate in the collection solution of white lupin roots. However, those compounds were found only in the following root segments of -P plants: juvenile (J), mature $(M)$, senescent $(S)$.

Citrate exudation was not affected by the type of root handling (Table 2). On the other hand, citrate exudation was influenced by root tissue type in the intact plant (IP) set-up. Juvenile and $M$ cluster roots both exuded significantly more citrate than senescent (S) rootlets (on average +94\%).

Malate exudation revealed a different pattern compared to citrate (Table 2). Especially, S rootlets showed a significant higher malate exudation when the plant was kept intact (IP), followed by the IR handling method and lastly the completely dissected roots (DR). The IP set-up in S rootlets showed a $90.6 \%$ higher malate release when compared to the IR set-up, while $237 \%$ higher with respect to the DR set-up. Malate exudation was affected by the root tissue type only in the IP handling set-up. The $S$ roots showed the highest malate release when compared to both $\mathrm{J}$ and $\mathrm{M}$ rootlets (+ $241 \% \mathrm{M}$ and $\mathrm{S}$ vs $\mathrm{J} ;+128 \% \mathrm{~S}$ vs $\mathrm{M}$ ).

Lactate exudation of white lupin roots $4 \mathrm{~h}$ after the onset of light was not altered significantly by the root handling methods (Table 2). On the other hand, the different root tissue types showed a significant effect on the lactate detected in the collection solution of the IR and DR roots. In the IR handling method, the J cluster roots displayed the significant highest release of lactate when compared to the senescent rootlets $(+109 \%$ J vs S). The same could be observed in the completely 
dissected roots handling method (DR). Juvenile cluster roots showed a $60 \%$ higher lactate release rate compared to $\mathrm{M}$ rootlets. It is interesting to note that no lactate was detected in the exudate collection solution of S rootlets in the DR handling method.

\section{Release of Carboxylates After $8 \mathrm{~h}$}

Table 3 reports the carboxylate exudation of both C and -P lupin plants detected in the collection solution of the different root segments after $8 \mathrm{~h}$ from onset of light. The carboxylate analysis of the exudate solutions revealed the presence of citrate, malate, and lactate in juvenile $(\mathrm{J})$, mature $(\mathrm{M})$ and senescent $(\mathrm{S})$ rootlets. Carboxylates were not detectable in the collection solutions of control (C) roots.

Citrate exudation of J roots was significantly altered by the root handling (Table 3). The completely dissecting of roots (DR) showed a significantly higher (91.3\%) citrate exudation rate compared to the set-up in which roots were cut only once (IR setup) and a $64.6 \%$ higher rate compared to intact plants (IP set-up). The same trend was observed in M rootlets (+56.2\% DR vs IR; $+95.9 \%$ DR $v s \mathrm{IP})$, as well as in S roots $(+175 \%$ DR vs IP). Citrate exudation was significantly affected by the different root segments within the same handling methods. In the IP- set-up, J rootlets exuded the significantly highest citrate rate when compared to both $\mathrm{M}$ and $\mathrm{S}$ cluster roots $(+75.5 \% \mathrm{~J}$ vs $\mathrm{M} ;+125 \% \mathrm{~J}$ vs $\mathrm{S})$. Furthermore, a similar trend could be observed in the handling method in which the roots were dissected completely into small segments (DR). Also, with this root handling method, the $\mathrm{J}$ rootlets displayed the significantly highest citrate exudation rate when compared to $\mathrm{M}$ cluster rootlets (+ 45\% J vs $\mathrm{M})$.

Malate exudation showed a completely different pattern than citrate exudation (Table 3). The root handling methods (IP, IR, $\mathrm{DR})$ revealed no significant effect on the malate exudation. However, in the set-up in which the plant was kept intact (IP) an effect of the different root tissue types could be observed. Very similar to citrate, malate release resulted highest in juvenile (J) cluster root compared to both mature $(\mathrm{M})$ and senescent $(\mathrm{S})$ rootlets $(+116 \% \mathrm{~J}$ vs $\mathrm{M},+105 \% \mathrm{~J}$ vs $\mathrm{S})$. No significant effect of the root tissue type was found in the other two handling methods (IR, DR). Interestingly, no malate was detected in the exudate collection solution of S rootlets in the completely dissected roots (DR).

Lactate exudation showed a similar trend as malate (Table 3). The root handling methods (IP, IR, DR) revealed no significant effect on the lactate exudation. On the other hand, an effect of the different root tissue types could be observed in the handling method in which lupin roots were dissected completely into pieces (DR). The J rootlets displayed an $82 \%$ higher exudation rate than M roots. Similarly, to malate, no lactate was detected in the exudate collection solution of $S$ rootlets in the completely dissected roots handling type (DR).

\section{DISCUSSION}

\section{Plant Morphology and General P Deficiency Symptoms}

This study used hydroponically grown white lupin plants together with a custom-made device (Figures 1 and 2) to examine how different root handling methods influenced both $\mathrm{P}_{\mathrm{i}}$ uptake and carboxylate exudation of single root segments when plants were subjected to either $\mathrm{P}$ sufficient or lacking conditions. The study used -P plants to enhance the root exudation and stimulate the development of cluster roots in white lupin. The concentration of $100 \mu \mathrm{mol} \mathrm{L}{ }^{-1} \mathrm{P}_{\mathrm{i}}$ was used to measure the potential capacity of $\mathrm{P}_{\mathrm{i}}$ uptake considering both low and high affinity $\mathrm{P}_{\mathrm{i}}$ transport systems (Furihata et al., 1992; Wang et al., 2017). The -P white lupins used in this research showed all the typical symptoms of $\mathrm{P}$ deficiency (Figure 3 ). Generally, well known symptoms of $\mathrm{P}$ deficiency in plants include an increased root-to-shoot ratio (López-Arredondo et al., 2014; Pandey et al., 2015), as well as alterations of the root morphology (Neumann and Martinoia, 2002; LópezArredondo et al., 2014). In fact, the -P plants in this study presented a $42 \%$ higher shoot to root ratio than $\mathrm{C}$ plants (Figure 3A). As expected, white lupin plants produced huge amounts of cluster roots to cope with P deficiency (Uhde-Stone, 2017) (Figure 3E). Cluster root formation is very complex and probably modulated by signaling hormones (e.g. auxin, nitric oxide), sucrose and miRNAs (e.g. miR399) (Liu and Vance, 2010; Wang et al., 2015; Uhde-Stone, 2017; Zhou et al., 2019). Additionally, cluster roots can be also formed in plants with sufficient $\mathrm{P}$ nutrition, although those cluster roots are not fully functional (Meng et al., 2013; Wang et al., 2015). Indeed, also this study was able to identify cluster root like structures in control plants (Figure 3D). Cluster roots do not only exude huge amounts of carboxylates but also intensely secret protons to solubilize the scarcely soluble $\mathrm{P}_{\mathrm{i}}$ (Wang et al., 2006; Uhde-Stone, 2017). Qualitative visualization of $\mathrm{pH}$ changes due to proton exudation was also performed in this study and confirms these findings. Indeed, Figure 3C shows that $-\mathrm{P}$ white lupin roots acidify (yellow coloration) the rhizosphere to a much greater extent compared to $\mathrm{C}$ white lupin roots (Figure 3B).

\section{Phosphate Uptake and Carboxylate Exudation}

A very important aspect in the context of this study is the presentation of the data, especially in terms of units (Oburger and Jones, 2018). The results were normalized (divided) by the roots' fresh weight. Normalization on root surface area or root length would completely bias the results since cluster roots of -P roots ( $\mathrm{M}, \mathrm{J}, \mathrm{S}$ rootlets) generally have a much higher fresh weight. Moreover, reproducible measurements of the roots surface area is complex and the literature generally recommends to normalize by the root weight (Oburger and Jones, 2018). On the other hand, since the weight of root tissue types such as $\mathrm{A}, \mathrm{R}$ and $\mathrm{AC}$ and $\mathrm{RC}$ is much smaller than the cluster roots the $\mathrm{P}_{\mathrm{i}}$ uptake/ carboxylate exudation is more pronounced when indicating the data per root fresh weight.

Since the aim of the study was to assess the difference between the root handling methods, one-way ANOVAs were chosen as statistical tests over pairwise comparisons. Even though pairwise comparisons could lead to more significant results, ANOVAS highlight the most relevant differences more suitable to the aim of the study. 


\section{Phosphate Uptake}

Phosphate uptake is a crucial process for plants. Compared to other major nutrients (such as nitrogen, potassium etc.), $\mathrm{P}_{\mathrm{i}}$ is far less mobile and thus more difficult for plants to acquire (Hinsinger, 2001). Especially when growing in P-deficient soils, plants must adapt in order to acquire all the available $\mathrm{P}_{\mathrm{i}}$. The measurement of plants' rhizosphere processes such as root $\mathrm{P}_{\mathrm{i}}$ uptake is methodologically very challenging (Oburger and Schmidt, 2016; Oburger and Jones, 2018), and even more so when targeting single root segments. Most studies which targeted root segments worked with excised roots (Vorster and Jooste, 1986; Massonneau et al., 2001) or with radioactive labeled ${ }^{32} \mathrm{P}$ (Kreuzwieser et al., 1997; Flatian et al., 2018; Cruz-Paredes and Gavito, 2020). Furthermore, there is a lot of research which used excised roots (Benlloch et al., 1983; Bloom and Caldwell, 1988; Tu et al., 1990; Rubio et al., 2004; Harley and McCready, 2006; Lucash et al., 2007; Abbas and Meharg, 2008; Horbowicz et al., 2011). Root handling methods such as dissecting/cutting/ excising/may disturb roots and thus artificially increase or decrease the net uptake (Bloom and Caldwell, 1988; Oburger and Schmidt, 2016). The present study revealed that the root handling influences the $\mathrm{P}_{\mathrm{i}}$ uptake rate of white lupin roots. However, the effect was limited to 4 and $8 \mathrm{~h}$ after the onset of light (Tables 2 and 3). No effect occurred $1 \mathrm{~h}$ after the onset of light (Table 1). White lupin plants are known to have a timedependent citrate exudation pattern (Dessureault-Rompré et al., 2007; Tomasi et al., 2009; Mimmo et al., 2011), releasing the main citrate burst around 4 to $5 \mathrm{~h}$ after the onset of light (Tomasi et al., 2009). Since the roots are probably more active at that time, it is most likely that the $\mathrm{P}_{\mathrm{i}}$ uptake was not influenced in the first sampling time point $(1 \mathrm{~h})$. Four hours after the onset of light, effects of the root handling methods on the $\mathrm{P}_{\mathrm{i}}$ uptake could be detected (Table 2), yet only in roots of plants grown in a full NS (AC, C, RC tissue). Roots completely dissected into pieces (DR) displayed in all three root tissue types the highest uptake rate. This indicates that the complete dissection increases the $\mathrm{P}_{\mathrm{i}}$ uptake greatly and can lead to biased data. A study conducted on cut maize roots with ${ }^{32} \mathrm{P}$ came to the same result, showing that the excision can enhance the $\mathrm{P}_{\mathrm{i}}$ influx (Gronewald and Hanson, 1980). In contrast to that, root dissection has been shown to decrease $\mathrm{NO}_{3}{ }^{-}$(Aslam et al., 1996) and $\mathrm{NH}_{4}{ }^{+}$uptake (Bloom and Caldwell, 1988). The sampling at $8 \mathrm{~h}$ after the onset of the light led to the most significant alterations of the root $P_{i}$ uptake (Table 3) induced by the sampling method. In some cases, a similar trend as at $4 \mathrm{~h}$ could be observed with the DR sampling method showing the highest uptake in most tissues (A, M, S, C, RC). Though, also the IP method showed a high $\mathrm{P}_{\mathrm{i}}$ uptake rate (Table 3). The dissection of the roots, both complete or just partial (IR, DR), has a huge impact and thus it could be expected to have a great influence on physiological parameters (e.g. $\mathrm{P}_{\mathrm{i}}$ uptake). Nevertheless, the influence of the root handling on $\mathrm{P}_{\mathrm{i}}$ uptake shows that the results should be carefully interpreted.

The A rootlet tissue type displayed in most cases the highest uptake when normalized per fresh weight. Therefore, it is supposed that the root apex/tip could act as a sensor in order to scout for nutrients. If no nutrients are sensed the tip triggers a signal cascade in order to cope with the nutrient deficiency, e.g. developing clusters and increasing exudation to enhance the availability of $\mathrm{P}_{\mathrm{i}}$. In fact, it has been already postulated by some authors in the case of Arabidopsis thaliana (Abel, 2017) and confirmed in maize (Zea mays L.) roots (Trevisan et al., 2015). Moreover, it might be hypothesized that root tips could sense concentration changes of exuded metabolites and translate this into signals to modify root growth (Canarini et al., 2019), enforcing the theory that the tip/apex of the root acts as a kind of sensor for the plant.

\section{Carboxylate Exudation}

Exudation of carboxylates represents one of the most common responses to nutrient deficiencies in plants (Vives-Peris et al., 2019). Exudation of carboxylates (citrate, malate and lactate) was affected significantly by the root handling method in the samplings 1 and $8 \mathrm{~h}$ after the onset of light (Tables 1 and 3 ). At $1 \mathrm{~h}$ after the onset of light the roots of intact plants (IP) had the tendency to exude the highest concentration of carboxylates (Table 1). In this case, the data suggest that the root dissection decreases the exudation rather than increasing it. Indeed, we rather hypothesized that the physical damage to the roots leads to a higher exudation. To the best of our knowledge, no scientific literature is available about the effect of root cutting/dissecting on root exudation. The only evidence about the effect of physical damage on root exudation is rather dated. These authors found that amino acids release from damaged wheat and pea roots leads to $73 \%$ to $120 \%$ of that released by intact roots (Ayers and Thornton, 1968). The sampling performed at $4 \mathrm{~h}$ after the onset of light had just minor effects on the exudation (Table 2). The lack of major significant alterations of the exudation rate at the 4-h sampling time point (at noon) could be explained again (as in section 4.2 .1 ) by the time-dependent citrate exudation pattern (Mimmo et al., 2011). The higher root activity at noon could counteract the disturbing effects of the root dissection. At $8 \mathrm{~h}$ after the onset of light only citrate exudation was affected by the root handling method (Table 3). However, the opposite trend was observed compared to the exudation at $4 \mathrm{~h}$ after the onset of light. The completely dissected roots showed the highest citrate exudation rate. For the first time, this study quantifies the effects of root handling/cutting/dissecting on the exudation of carboxylates. Although no clear and distinctive trend could be observed, we revealed that the handling and the sampling time point can greatly influence the outcomes. Undeniably, these two factors should not be neglected when interpreting rhizosphere dynamics.

\section{CONCLUSION}

The present study examined for the first time the effect of three different root handling methods on the $\mathrm{P}_{\mathrm{i}}$ uptake and carboxylate exudation of different cluster root zones of white lupin plants grown hydroponically in a full and P-deficient nutrient solution. The results imply that the handling method influences greatly both $\mathrm{P}_{\mathrm{i}}$ uptake and carboxylate exudation and thus has to be 
considered when studying roots in a destructive way. Moreover, the study revealed, that in plant species like white lupins, which shows a diurnal pattern of root activity, the effect of the root handling depends on sampling time point. Additionally, the study enforced the growing evidence that the root tip acts as a sensor for $\mathrm{P}_{\mathrm{i}}$.

\section{DATA AVAILABILITY STATEMENT}

The raw data supporting the conclusions of this article will be made available by the authors, without undue reservation.

\section{AUTHOR CONTRIBUTIONS}

TM, StC, YP, and RT designed the study. RT, FV, and $\mathrm{SiC}$ performed the experiments. RT, TM, and StC wrote the paper.

\section{REFERENCES}

Abbas, M. H. H., and Meharg, A. A. (2008). Arsenate, arsenite and dimethyl arsinic acid (DMA) uptake and tolerance in maize (Zea mays L.). Plant Soil 304, 277-289. doi: 10.1007/s11104-008-9549-9

Abel, S. (2017). Phosphate scouting by root tips. Curr. Opin. Plant Biol. 39, 168177. doi: 10.1016/j.pbi.2017.04.016

Aslam, M., Travis, R. L., Rains, D. W., and Huffaker, R. C. (1996). Effect of root perturbation and excision on nitrate influx and efflux in barley (Hordeum vulgare) seedlings. Physiol. Plant 97, 425-432. doi: 10.1111/j.13993054.1996.tb00499.x

Ayers, W. A., and Thornton, R. H. (1968). Exudation of amino acids by intact and damaged roots of wheat and peas. Plant Soil 28, 193-207. doi: 10.1007/ BF01880238

Baetz, U., and Martinoia, E. (2014). Root exudates: the hidden part of plant defense. Trends Plant Sci. 19, 90-98. doi: 10.1016/j.tplants.2013.11.006

Benlloch, M., Fournier, J. M., and de la Guardia, M. D. (1983). Effect of gibberellic acid on $\mathrm{K}+(\mathrm{Rb}+)$ uptake and transport in sunflower roots. Physiol. Plant 57, 79-84. doi: 10.1111/j.1399-3054.1983.tb00733.x

Bloom, A. J., and Caldwell, R. M. (1988). Root Excision Decreases Nutrient Absorption and Gas Fluxes. Plant Physiol. 87, 794 LP - 796. doi: 10.1104/ pp.87.4.794

Canarini, A., Kaiser, C., Merchant, A., Richter, A., and Wanek, W. (2019). Root exudation of primary metabolites: Mechanisms and their roles in plant responses to environmental stimuli. Front. Plant Sci. 10, 157-176. doi: $10.3389 /$ fpls.2019.00157

Cruz-Paredes, C., and Gavito, M. E. (2020). Isotope Labeling to Study Phosphorus Uptake in the Arbuscular Mycorrhizal Symbiosis BT - Arbuscular Mycorrhizal Fungi: Methods and Protocols. Eds. N. Ferrol and L. Lanfranco (New York, NY: Springer US), 213-222. doi: 10.1007/978-1-0716-0603-2_16

Cruz-Ramírez, A., Oropeza-Aburto, A., Razo-Hernández, F., Ramírez-Chávez, E., and Herrera-Estrella, L. (2006). Phospholipase DZ2 plays an important role in extraplastidic galactolipid biosynthesis and phosphate recycling in Arabidopsis roots. Proc. Natl. Acad. Sci. 103, 6765 LP - 6770. doi: 10.1073/ pnas.0600863103

Dessureault-Rompré, J., Nowack, B., Schulin, R., and Luster, J. (2007). Spatial and temporal variation in organic acid anion exudation and nutrient anion uptake in the rhizosphere of Lupinus albus L. Plant Soil 301, 123-134. doi: 10.1007/ s11104-007-9427-x

Dinkelaker, B., Römheld, V., and Marschner, H. (1989). Citric acid excretion and precipitation of calcium citrate in the rhizosphere of white lupin (Lupinus albus L.). Plant Cell Environ. 12, 285-292. doi: 10.1111/j.1365-3040.1989. tb01942.x
All authors contributed to the article and approved the submitted version.

\section{FUNDING}

This work was supported by grants from the Free University of Bolzano (NUMICS TN200E). The doctoral fellowship of RT was funded by Stiftung Südtiroler Sparkasse.

\section{ACKNOWLEDGMENTS}

We would like to thank Markus Fischnaller and the team from the BITZ unibz fablab for the support and the 3D print of the custom-made devices used in this study. Furthermore, we would like to thank prof Nicola Tomasi from the University of Udine for the valuable discussion.

Flatian, A. N., Slamet, S., and Citraresmini, A. (2018). Phosphorus (P) uptake by sorghum plant derived from 2 types of $\mathrm{p}$ fertilizers traced by isotopic technique (32p). J. Ilm. Apl. Isot. dan Radiasi 14, 109-115.

Fredeen, A. L., Rao, I. M., and Terry, N. (1989). Influence of Phosphorus Nutrition on Growth and Carbon Partitioning in Glycine max. Plant Physiol. 89, 225 LP -230. doi: 10.1104/pp.89.1.225

Furihata, T., Suzuki, M., and Sakurai, H. (1992). Kinetic Characterization of Two Phosphate Uptake Systems with Different Affinities in Suspension-Cultured Catharanthus roseus Protoplasts. Plant Cell Physiol. 33, 1151-1157. doi: 10.1093/oxfordjournals.pcp.a078367

Gaude, N., Nakamura, Y., Scheible, W.-R., Ohta, H., and Dörmann, P. (2008). Phospholipase C5 (NPC5) is involved in galactolipid accumulation during phosphate limitation in leaves of Arabidopsis. Plant J. 56, 28-39. doi: 10.1111/ j.1365-313X.2008.03582.x

Gronewald, J. W., and Hanson, J. B. (1980). Sensitivity of the proton and ion transport mechanisms of corn roots to injury. Plant Sci. Lett. 18, 143-150. doi: 10.1016/0304-4211(80)90043-7

Harley, J., and McCready, C. (2006). The uptake of phosphate by excised mycorrhizal roots of the beech. New Phytol. 51, 56-64. doi: 10.1111/j.14698137.1952.tb06115.x

Hinsinger, P. (2001). Bioavailability of soil inorganic P in the rhizosphere as affected by root-induced chemical changes: A review. Plant Soil 237, 173-195. doi: 10.1023/A:1013351617532

Horbowicz, M., Kowalczyk, W., Grzesiuk, A., and Mitrus, J. (2011). Uptake of aluminium and basic elements, and accumulation of anthocyanins in seedlings of common buckwheat (Fagopyrum esculentum moench) as a result increased level of aluminium in nutrient solution. Ecol. Chem. Eng. S. 18, 479-488. https://www. scopus.com/inward/record.uri?eid=2-s2.0-84860662117\&partnerID=40\&md5 = aa856b86481e9c38630440b1fa912864.

Jones, D. L., Nguyen, C., and Finlay, R. D. (2009). Carbon flow in the rhizosphere: carbon trading at the soil-root interface. Plant Soil 321, 5-33. doi: 10.1007/ s11104-009-9925-0

Kreuzwieser, J., Herschbach, C., Stulen, I., Wiersema, P., Vaalburg, W., and Rennenberg, H. (1997). Interactions of NH4+ and L-glutamate with NO3transport processes of non-mycorrhizal Fagus sylvatica roots. J. Exp. Bot. 48, 1431-1438. doi: 10.1093/jxb/48.7.1431

Lambers, H., Shane, M. W., Cramer, M. D., Pearse, S. J., and Veneklaas, E. J. (2006). Root Structure and Functioning for Efficient Acquisition of Phosphorus: Matching Morphological and Physiological Traits. Ann. Bot. 98, 693-713. doi: 10.1093/aob/mcl114

Lamont, B. (1982). Mechanisms for enhancing nutrient uptake in plants, with particular reference to mediterranean South Africa and Western Australia. Bot. Rev. 48, 597-689. doi: 10.1007/BF02860714 
Liu, J., and Vance, C. P. (2010). Crucial roles of sucrose and microRNA399 in systemic signaling of P deficiency: a tale of two team players? Plant Signal. Behav. 5, 1556-1560. doi: 10.4161/psb.5.12.13293

López-Arredondo, D. L., Leyva-González, M. A., González-Morales, S.II, LópezBucio, J., and Herrera-Estrella, L. (2014). Phosphate Nutrition: Improving Low-Phosphate Tolerance in Crops. Annu. Rev. Plant Biol. 65, 95-123. doi: 10.1146/annurev-arplant-050213-035949

Lucash, M. S., Eissenstat, D. M., Joslin, J. D., McFarlane, K. J., and Yanai, R. D. (2007). Estimating nutrient uptake by mature tree roots under field conditions: Challenges and opportunities. Trees - Struct. Funct. 21, 593-603. doi: 10.1007/ s00468-007-0160-0

Malajczuk, N., and Bowen, G. D. (1974). Proteoid roots are microbially induced. Nature 251, 316-317. doi: 10.1038/251316a0

Marschner, P. (2012). Mineral Nutrition of Higher Plants. (Marschner: Elsevier, Academic Press, Uhde-Stone: Springer). doi: 10.1016/B978-0-12-384905-2.00013-3

Massonneau, A., Langlade, N., Léon, S., Smutny, J., Vogt, E., Neumann, G., et al. (2001). Metabolic changes associated with cluster root development in white lupin (Lupinus albus L.): relationship between organic acid excretion, sucrose metabolism and energy status. Planta 213, 534-542. doi: 10.1007/ s004250100529

Meng, Z.B., You, X. D., Suo, D., Chen, Y. L., Tang, C., Yang, J. L., et al. (2013). Root-derived auxin contributes to the phosphorus-deficiency-induced clusterroot formation in white lupin (Lupinus albus). Physiol. Plant 148, 481-489. doi: 10.1111/j.1399-3054.2012.01715.x

Mimmo, T., Hann, S., Jaitz, L., Cesco, S., Gessa, C. E., and Puschenreiter, M. (2011). Time and substrate dependent exudation of carboxylates by Lupinus albus L. and Brassica napus L. Plant Physiol. Biochem. 49, 1272-1278. doi: 10.1016/j.plaphy.2011.08.012

Müller, J., Gödde, V., Niehaus, K., and Zörb, C. (2015). Metabolic Adaptations of White Lupin Roots and Shoots under Phosphorus Deficiency. Front. Plant Sci. 6, 1014. doi: 10.3389/fpls.2015.01014

Murphy, J., and Riley, J. P. (1962). A modified single solution method for the determination of phosphate in natural waters. Anal. Chim. Acta 27, 31-36. doi: $10.1016 /$ S0003-2670(00)88444-5

Neumann, G., and Martinoia, E. (2002). Cluster roots - an underground adaptation for survival in extreme environments. Trends Plant Sci. 7, 162167. doi: $10.1016 / S 1360-1385(02) 02241-0$

Neumann, G., and Römheld, V. (1999). Root excretion of carboxylic acids and protons in phosphorus-deficient plants. Plant Soil 211, 121-130. doi: 10.1023/ A:1004380832118

Oburger, E., and Jones, D. L. (2018). Sampling root exudates - Mission impossible? Rhizosphere 6, 116-133. doi: 10.1016/j.rhisph.2018.06.004

Oburger, E., and Schmidt, H. (2016). New Methods To Unravel Rhizosphere Processes. Trends Plant Sci. 21, 243-255. doi: 10.1016/j.tplants.2015.12.005

Otani, T., Ae, N., and Tanaka, H. (1996). Phosphorus (P) Uptake mechanisms of crops grown in soils with low P status. Soil Sci. Plant Nutr. 42, 553-560. doi: $10.1080 / 00380768.1996 .10416324$

Pandey, R., Zinta, G., AbdElgawad, H., Ahmad, A., Jain, V., and Janssens, I. A. (2015). Physiological and molecular alterations in plants exposed to high [CO2] under phosphorus stress. Biotechnol. Adv. 33, 303-316. doi: 10.1016/ j.biotechadv.2015.03.011

Pausch, J., and Kuzyakov, Y. (2018). Carbon input by roots into the soil: Quantification of rhizodeposition from root to ecosystem scale. Glob. Change Biol. 24, 1-12. doi: 10.1111/gcb.13850

Péret, B., Clément, M., Nussaume, L., and Desnos, T. (2011). Root developmental adaptation to phosphate starvation: better safe than sorry. Trends Plant Sci. 16, 442-450. doi: 10.1016/j.tplants.2011.05.006

Raghothama, K. G. (1999). Phosphate acquisition. Annu. Rev. Plant Physiol. Plant Mol. Biol. 50, 665-693. doi: 10.1146/annurev.arplant.50.1.665

Reymond, M., Svistoonof, S., Loudet, O., Nussaume, L., and Desnos, T. (2006). Identification of QTL controlling root growth response to phosphate starvation in Arabidopsis thaliana. Plant Cell Environ. 29, 115-125. doi: 10.1111/j.13653040.2005.01405.x

Rodríguez, D., Keltjens, W. G., and Goudriaan, J. (1998). Plant leaf area expansion and assimilate production in wheat (Triticum aestivum L.) growing under low phosphorus conditions. Plant Soil 200, 227-240. doi: 10.1023/A:1004310 217694
Rubio, G., Sorgonà, A., and Lynch, J. P. (2004). Spatial mapping of phosphorus influx in bean root systems using digital autoradiography. J. Exp. Bot. 55, 22692280. doi: $10.1093 / \mathrm{jxb} / \mathrm{erh} 246$

Shane, M. W., Cramer, M. D., Funayama-Noguchi, S., Cawthray, G. R., Millar, A. H., Day, D. A., et al. (2004). Developmental Physiology of Cluster-Root Carboxylate Synthesis and Exudation in Harsh Hakea. Expression of Phosphoenopyruvate Carboxylase and the Alternative Oxidase. Plant Physiol. 135, 549 LP - 560. doi: 10.1104/pp.103.035659

Skene, K. R. (2000). Pattern Formation in Cluster Roots: Some Developmental and Evolutionary Considerations. Ann. Bot. 85, 901-908. doi: 10.1006/ anbo.2000.1140

Steinauer, K., Chatzinotas, A., and Eisenhauer, N. (2016). Root exudate cocktails: the link between plant diversity and soil microorganisms? Ecol. Evol. 6, 73877396. doi: $10.1002 /$ ece3.2454

Tomasi, N., Weisskopf, L., Renella, G., Landi, L., Pinton, R., Varanini, Z., et al. (2008). Flavonoids of white lupin roots participate in phosphorus mobilization from soil. Soil Biol. Biochem. 40, 1971-1974. doi: 10.1016/j.soilbio.2008.02.017

Tomasi, N., Kretzschmar, T., Espen, L., Weisskopf, L., Fuglsang, A. T., Palmgren, M. G., et al. (2009). Plasma membrane H-ATPase-dependent citrate exudation from cluster roots of phosphate-deficient white lupin. Plant Cell Environ. 32, 465-475. doi: 10.1111/j.1365-3040.2009.01938.x

Tomasi, N., De Nobili, M., Gottardi, S., Zanin, L., Mimmo, T., Varanini, Z., et al. (2013). Physiological and molecular characterization of $\mathrm{Fe}$ acquisition by tomato plants from natural Fe complexes. Biol. Fertil. Soils 49, 187-200. doi: $10.1007 / \mathrm{s} 00374-012-0706-1$

Trevisan, S., Manoli, A., Ravazzolo, L., Botton, A., Pivato, M., Masi, A., et al. (2015). Nitrate sensing by the maize root apex transition zone: a merged transcriptomic and proteomic survey. J. Exp. Bot. 66, 3699-3715. doi: 10.1093/ jxb/erv165

Tu, S.II, Cavanaugh, J. R., and Boswell, R. T. (1990). Phosphate Uptake by Excised Maize Root Tips Studied by in VivoP Nuclear Magnetic Resonance Spectroscopy. Plant Physiol. 93, 778-784. doi: 10.1104/pp.93.2.778

Turnbull, T. L., Warren, C. R., and Adams, M. A. (2007). Novel mannosesequestration technique reveals variation in subcellular orthophosphate pools do not explain the effects of phosphorus nutrition on photosynthesis in Eucalyptus globulus seedlings. New Phytol. 176, 849-861. doi: 10.1111/ j.1469-8137.2007.02229.x

Uhde-Stone, C. (2017). "White Lupin: A Model System for Understanding Plant Adaptation to Low Phosphorus Availability," in Legume Nitrogen Fixation in Soils with Low Phosphorus Availability: Adaptation and Regulatory Implication (Springer), 243-280. doi: 10.1007/978-3-319-55729-8_13

Valentinuzzi, F., Cesco, S., Tomasi, N., and Mimmo, T. (2015). Influence of different trap solutions on the determination of root exudates in Lupinus albus L. Biol. Fertil. Soils 51, 757-765. doi: 10.1007/s00374-015-1015-2

van de Wiel, C. C. M., van der Linden, C. G., and Scholten, O. E. (2016). Improving phosphorus use efficiency in agriculture: opportunities for breeding. Euphytica 207, 1-22. doi: 10.1007/s10681-015-1572-3

Veneklaas, E. J., Lambers, H., Bragg, J., Finnegan, P. M., Lovelock, C. E., Plaxton, W. C., et al. (2012). Opportunities for improving phosphorus-use efficiency in crop plants. New Phytol. 195, 306-320. doi: 10.1111/j.1469-8137.2012.04190.x

Vives-Peris, V., de Ollas, C., Gómez-Cadenas, A., and Pérez-Clemente, R. M. (2019). Root exudates: from plant to rhizosphere and beyond. Plant Cell Rep. 39, 3-17. doi: 10.1007/s00299-019-02447-5

Vorster, P. W., and Jooste, J. H. (1986). Potassium and phosphate absorption by excised ordinary and proteoid roots of the Proteaceae. South Afr. J. Bot. 52, 277-281. doi: 10.1016/S0254-6299(16)31520-4

Wang, Z., Shen, J., and Zhang, F. (2006). Cluster-root formation, carboxylate exudation and proton release of Lupinus pilosus Murr. as affected by medium $\mathrm{pH}$ and P deficiency. Plant Soil 287, 247-256. doi: 10.1007/s11104-006-9071-x

Wang, Z., Straub, D., Yang, H., Kania, A., Shen, J., Ludewig, U., et al. (2014). The regulatory network of cluster-root function and development in phosphatedeficient white lupin (Lupinus albus) identified by transcriptome sequencing. Physiol. Plant 151, 323-338. doi: 10.1111/ppl.12187

Wang, Z., Shen, J., Ludewig, U., and Neumann, G. (2015). A re-assessment of sucrose signaling involved in cluster-root formation and function in phosphate-deficient white lupin (Lupinus albus). Physiol. Plant 154, 407419. doi: 10.1111/ppl.12311 
Wang, D., Lv, S., Jiang, P., and Li, Y. (2017). Roles, regulation, and agricultural application of plant phosphate transporters. Front. Plant Sci. 8, 817. doi: $10.3389 /$ fpls.2017.00817

Weisskopf, L., Tomasi, N., Santelia, D., Martinoia, E., Langlade, N. B., Tabacchi, R., et al. (2006). Isoflavonoid exudation from white lupin roots is influenced by phosphate supply, root type and cluster-root stage. New Phytol. 171, 657-668. doi: 10.1111/j.1469-8137.2006.01776.x

Wissuwa, M., Gamat, G., and Ismail, A. M. (2005). Is root growth under phosphorus deficiency affected by source or sink limitations? J. Exp. Bot. 56, 1943-1950. doi: 10.1093/jxb/eri189

Zhou, Y., Sarker, U., Neumann, G., and Ludewig, U. (2019). The LaCEP1 peptide modulates cluster root morphology in Lupinus albus. Physiol. Plant 166, 525537. doi: 10.1111/ppl.12799
Conflict of Interest: The authors declare that the research was conducted in the absence of any commercial or financial relationships that could be construed as a potential conflict of interest.

The handling Editor declared a past co-authorship with three of the authors, TM, $\mathrm{SiC}$, and $\mathrm{StC}$.

Copyright (C) 2020 Tiziani, Mimmo, Valentinuzzi, Pii, Celletti and Cesco. This is an open-access article distributed under the terms of the Creative Commons Attribution License (CC BY). The use, distribution or reproduction in other forums is permitted, provided the original author(s) and the copyright owner(s) are credited and that the original publication in this journal is cited, in accordance with accepted academic practice. No use, distribution or reproduction is permitted which does not comply with these terms. 